each other, blood tests. Final diagnosis was determined by the third US expert if the 2 observers got the different conclusion. Bilateral toes, dorsal feet, ankle, knee, wrist, fingers, elbow, and shoulder were detected to find 6 features of gout suggested by OMERCT: tophus, "snow storm"-like effusion, cloudy synovium in grey scale, double-contour sign, bone erosion and Power Doppler (PD) signal. Each above positive presentations in each above range would get 1 point and the sum scores would be ranged from 0 to 84 . Serum uric acid (UA) was recorded too.

Results: 1) 23 female and 139 male were recruited in the program. The femalemale ratio was 1:6. Mean age and disease duration of the female subjects were elder than male ones (female:male $=57.2 \pm 14.1: 44.7 \pm 14.7$ years old) with longer disease duration to confim the diagnosis (female:male $=10.9: 1.2$ months). The average serum UA level in female was lower than male group (female:male = $413.8 \pm 162.1 \mathrm{umol} / \mathrm{L}, 515.5 \pm 156.9 \mathrm{umol} / \mathrm{L}, \mathrm{sig}<0.05)$.

2) The intra-observer reliability from $20 \%$ samples random seclection showed an overall agreement of $80 \%, 92 \%, 96 \%, 87 \%, 80 \%, 73 \%$ for tophus, "snow storm"-like effusion, cloudy synovium in grey scale, positive double-contour sign, bone erosion and PD signal with kappa value of $0.78,0.92,0.95,0.86,0.79$, 0.72 , respectively.

3) The difference showed female gout had higher frequency of tophus, bone erosion and lower frequency of effusion while the other indexes were equal:topus scores (female:male=87\%:,74.1\%), cloudy synovium grey scale scores (female:male $=65.2 \%: 62.6 \%$ ), effusion scores (female:male $=17.4 \%, 31.7 \%$ ), bone erosion scores (female:male $=30.4 \%, 16.5 \%$ ), power dopplar scores (female:male $=34.8 \%, 41 \%)$, positive double-contour signs $(60.9 \%: 59.9 \%$. The top 2 affected ranges were ankle (female:male $=69.6 \%: 55.3 \%$ ), knee (female:mlae $=60.9 \%: 54.0 \%)$. Furthermore, female gout had more frequently occurred not in the less typical ranges such as fingers (female:male $=34.7 \%: 19.4 \%$ ), elbow (8.7\%:2.7\%), which might be the cause for delayed diagnosis.

Conclusions: Though the level of serum UA was lower, Female gout had its unique ultrasound features with more tophus, bone erosion and less effusion compared to male gout. The less typical ranges were recommended for US examinations.

Disclosure of Interest: None declared

DOI: 10.1136/annrheumdis-2017-eular.2636

\section{THU0422 SEL-212: ENHANCED SERUM URIC ACID CONTROL IN HYPERURICEMIC PATIENTS THROUGH SELECTIVE MITIGATION OF ANTI-DRUG ANTIBODIES AGAINST PEGSITICASE}

E. Sands ${ }^{1}$, A. Kivitz ${ }^{2}$, L. Johnston ${ }^{1}$, T.K. Kishimoto ${ }^{1} .{ }^{1}$ Selecta Biosciences, Watertown; ${ }^{2}$ Altoona Center for Clinical Research, Duncansville, United States

Background: Recent EULAR recommendations for refractory gout treatment with pegylated uricase (pegloticase) acknowledge the risk of allergic reactions related to the development of anti-drug antibodies (ADAs) [1]. ADAs also affect the efficacy of treatment [2]. As a novel approach to treatment, we demonstrated that co-administration of pegsiticase (another pegylated uricase) and a synthetic vaccine particle encapsulating rapamycin (SVP-R) showed improved control of serum uric acid (sUA) in uricase-deficient mice by inducing antigen-specific immune tolerance to pegsiticase [3]. Here we describe the impact of SEL-212, a combination product of SVP-R and pegsiticase, on ADA formation and SUA levels in hyperuricemic patients in a Phase 1 open-label multicenter clinical trial.

Objectives: To assess the initial safety and impact on sUA levels and ADA formation of SEL-212, which is designed to be the first non-immunogenic uricase therapy for refractory gout.

Methods: Cohorts of hyperuricemic (sUA $>6 \mathrm{mg} / \mathrm{dL}$ ) patients consented to a single dose of $0.4 \mathrm{mg} / \mathrm{kg}$ pegsiticase alone, SVP-R alone $(0.03-0.5-\mathrm{mg} / \mathrm{kg})$, or $0.4 \mathrm{mg} / \mathrm{kg}$ pegsiticase co-administered with SVP-R (0.03-0.3-mg/kg; SEL-212). ADAs and SUA were assessed at baseline and $7,14,21$, and 30 days after dosing.

Results: Sixty-three patients were enrolled with a median age of 49.4 years. Mean baseline sUA was $7.4 \pm 1.3 \mathrm{mg} / \mathrm{dL}$. Patients dosed with pegsiticase alone showed an immediate drop in sUA, which returned to baseline levels by 14-21 days in 4 of 5 subjects, correlating with the induction of ADA titers $>1000$. Patients treated with SVP-R alone showed no meaningful change in SUA.

In contrast, patients treated with SEL-212 showed a dose-dependent inhibition of anti-uricase ADAs and corresponding decrease in sUA levels through at least day 30 after a single injection. Seven of 10 patients treated with SEL-212 at a SVP-R dose of $0.1 \mathrm{mg} / \mathrm{kg}$ showed no detectable sUA at day 30 , and all 10 subjects dosed with SEL-212 at SVP-R doses of 0.15 or $0.3 \mathrm{mg} / \mathrm{kg}$ showed sustained control of sUA through at least day 30 . There was a strong correlation between maintenance of low uric acid levels at day 30 and with low or no ADA titers.

SEL-212 was generally well tolerated at effective dose levels. One SAE (grade 2 rash) was observed in the lowest of the three effective dose levels $(0.1 \mathrm{mg} / \mathrm{kg}$ SVP-R). A second SAE was determined by the investigator to be not related to study drug. All SAEs fully resolved. No SAEs were observed with SEL-212 at the higher effective dose levels of SVP-R ( 0.15 or $0.3 \mathrm{mg} / \mathrm{kg}$ ). The maximum tolerated dose was defined at $0.3 \mathrm{mg} / \mathrm{kg}$.

Conclusions: Data suggest that a single dose of SEL-212 in hyperuricemic patients can tolerably, therapeutically and durably control sUA for $\geq 30$ days, correlating with inhibition of ADAs. These results supported monthly dosing in an ongoing Phase 2 multi-dose study in symptomatic gout patients and the potential use of SVP-R to mitigate ADAs for other immunogenic biologics.

\section{References:}

[1] Richette P, et al., 2017, 76:29-42.

[2] Lipsky PE, et al., Arthritis Res Ther. 2014, 16:R60.

[3] Kishimoto, TK, et al., Nat Nanotechnol. 2016, 11:890-899.

Disclosure of Interest: E. Sands Employee of: CMO for Selecta Biosciences, A. Kivitz: None declared, L. Johnston Employee of: $\mathrm{COO}$ for Selecta Biosciences, T. K. Kishimoto Employee of: CSO for Selecta Biosciences

DOI: 10.1136/annrheumdis-2017-eular.3548

\section{THU0423 THE RELATIONSHIP BETWEEN CLINICAL DISEASE ACTIVITY, SYMPTOM DURATION AND ULTRASONOGRAPHIC CHANGES IN GOUT}

E. Norkuviene ${ }^{1}$, M. Petraitis ${ }^{1}$, I. Apanaviciene ${ }^{1}$, D. Virviciute ${ }^{2}$,

A. Baranauskaite ${ }^{1} .{ }^{1}$ Rheumatology; ${ }^{2}$ Institute of Cardiology, Lithuanian

University of Health Sciences, Kaunas, Lithuania

Background: Ultrasonography (US) has recently been validated and used as an objective diagnostic tool for urate deposition also joint damage and was proposed as an outcome measure in gout [1-2].

Objectives: Our aim was to investigate the relationship between clinical gout activity and load of US changes.

Methods: Sixty monosodium-urate-crystal-confirmed gout patients (52 men) and 36 healthy normouricemic controls were prospectively included in one centre. The relationship between clinical symptoms and US gout-related changes investigating 36 joints and 4 tendons ( $m$. triceps and patellar) was evaluated using Spearman's correlation.

Results: The total number of intraarticular T, periarticular T, total T, total DC, and total $E$ found per patient on ultrasound ranged from $0-26,0-4,0-30,0-29$, and $0-18$, accordingly. The number of acute attacks per year/per life had a significant positive correlation with the total number of intraarticular $T$ ( $r s=0.518 / 0.652$; $p<0.0001)$, total number of intraarticular and periarticular $T$ ( $r s=0.552 / 0.699$; $p<0.0001)$, the total number of $D C(r s=0.374 / 0.551 ; p<0.01)$, the total number of erosions ( $r s=0.374 / 0.542 ; p<0.01$ ), and the total tophus area $(r s=0.420 / 0.549$; $p<0.01$ ) measured on US per patient. Strong, positive correlation was observed between the number of subcutaneous tophi and total US tophus area $(\mathrm{r} s=0.628)$, total number of $D C(r s=0.612)$ and erosions $(r s=0.526)$, found per patient on US, all $p<0.0001$. Disease duration significantly positively correlated with the load of US T, E and DC $(p<0.0001)$ in the investigated sites. There was no correlation between CRP and US, also no correlation between the uric acid concentration and US changes: total number of $T(r s=0.193, p=0.139), D C(r s=0.179, p=0.170)$ or $E(r s=0.063, p=0.634)$ found per patient. The tophus area measured in two first metatarsophalangeal joints (MTP) positively correlated with subcutaneous tophus count ( $r S=0,404 ; p=0,001)$, the US intraarticular T count ( $r s=0,732 ; p<0,0001$ ) total US DC count ( $r s=0,477 ; p<0,0001$ ) total intraarticular tophus area ( $r s$ $=0,829 ; p<0,0001$ ) and total tophus area other than first MTP joints ( $r s=0,603$; $p<0.0001$.

Conclusions: Ultrasonographic gout -related changes strongly positively correlate between each other and with subjective also objective signs of disease activity, increasing with disease duration in gout. The size of tophi inside the first metatarsophalangeal joints could be representative of the total body urate load and could be chosen as an outcome measure for the longitudinal gout studies. References:

[1] Neogi T, Jansen TLTA, Dalbeth N, Fransen J, Schumacher HR, Berendsen D, et al. 2015 Gout classification criteria: an American College of Rheumatology/European League Against Rheumatism collaborative initiative. Ann Rheum Dis. 2015 Sep 9; 74(10):1789-98.

[2] Ottaviani S, Gill G, Aubrun A, Palazzo E, Meyer O, Dieudé P. Ultrasound in gout: a useful tool for following urate-lowering therapy. Jt Bone Spine 2015 Jan; 82(1):42-4.

Disclosure of Interest: None declared

DOI: 10.1136/annrheumdis-2017-eular.6466

\section{THU0424 GOUT AT THE SPINE: A RETROSPECTIVE STUDY WITH DUAL-ENERGY COMPUTED TOMOGRAPHY}

E. Chotard $^{1}$, J.M. Sverzut ${ }^{2}$, F. Lioté ${ }^{1,3}$, T. Bardin ${ }^{1,3}$, H.-K. Ea ${ }^{1,3} .{ }^{1}$ AP-HP, hôpital Lariboisière, Service de Rhumatologie, centre Viggo Petersen, Paris; ${ }^{2}$ Centre cardiologique du Nord, Service de Radiologie, St Ouen; ${ }^{3}$ INSERM UMR1132, Bioscar, University Paris Diderot, Paris, France

Background: Gout is due to monosodium urate (MSU) crystal deposition after chronic hyperuricemia. Although MSU crystal deposition can occur in every joint and peri-articular structure, spine involvement is scarcely reported. Dual energy computed tomography (DECT) is a performant tool to assess urate deposits, especially in deep structures such as intervertebral discs and apophyseal joints. Objectives: to describe spinal DECT features of urate monosodium deposits compared to peripheral joint DECT.

Methods: Patient with gout diagnosis (MSU crystal identification by polarized microscopy or fulfilling "Nijmegen's criteria" (1)) who had undergone DECT were included from November 2012 to June 2016. Images were analyzed by 
a trained musculoskeletal radiologist. For each DECT, clinical and biochemical characteristics of each patient were collected retrospectively.

Results: 22 patients (men 77\%), mean age 62.5 years and mean BMI 28.4 $\mathrm{kg} / \mathrm{m}^{2}$ were included. Mean gout duration was $108.0 \pm 114.4$ months, mean of last available serum uric acid level was $520 \pm 193 \mu \mathrm{mol} / \mathrm{l}$, and 15 patients had at least one clinical tophus. Mean estimated glomerular filtration rate (MDRD formula) was $47 \pm 27 \mathrm{ml} / \mathrm{min} / 1.73 \mathrm{~m}^{2}$. One patient was on hemodialysis and one had received kidney transplant.

A total of 39 DECT has been performed: 28 of peripheral joints and 11 of the spine (9 lumbar, 1 sacroiliac and 1 cervical). Spinal DECT were done in 10 patients to explore recurrent inflammatory pain $(n=3$ lumbar, 1 cervical and 1 buttock) or mechanical back pain ( $n=2$ lumbar). 4 spinal DECT were performed in asymptomatic patients with extended peripheral tophi. Spinal MSU crystal deposits were disclosed by DECT in $83 \%(5 / 6)$ and $25 \%$ (1/4) of symptomatic and asymptomatic patients, respectively. In all painful patients, MSU crystal deposition was considered as a likely explanation of spinal symptoms. MSU crystal depositions was identified in apophyseal joints $(n=5)$, cervical intervertebral disc $(n=1)$ and yellow or interspinous ligaments $(n=4)$. All involved apophyseal joints were eroded (figure 1). No vertebral bone erosion was observed. Calcification of spinal tophus was observed in 4 patients. DECT identified peripheral deposits in $15 / 18(83.3 \%)$ patients. In peripheral DECT, bone erosions were observed in $71.4 \%$ and joint effusion in $32.1 \%$ of DECT positive peripheral joints. MSU crystal depositions were observed in tendons, cartilages or synovial membranes in $82.1 \%$ of positive DECT joints and in soft tissues in $64.3 \%$ of positive patients. MSU crystal deposits were calcified in 7 cases.

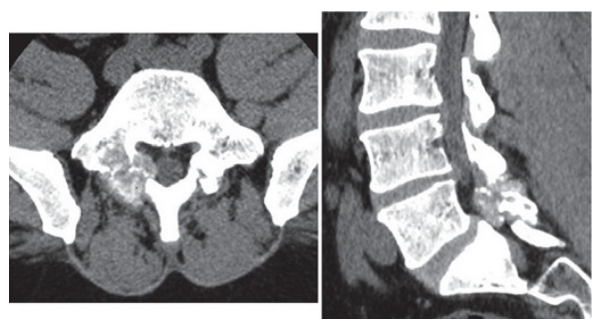

Conclusions: MSU crystal depositions at the spine are present in $60 \%$ of patients in this retrospective DECT study. DECT can represent a performing imaging procedure for their detection in symptomatic patients. Further studies are needed to assess the clinical utility of DECT of the spine in gout.

References:

[1] Janssens HJ, Fransen J, van de Lisdonk EH, et al. A diagnostic rule for acute gouty arthritis in primary care without joint fluid analysis. Arch Intern Med 2010;170:1120-6.

Disclosure of Interest: None declared

DOI: 10.1136/annrheumdis-2017-eular.6835

\section{THU0425 THE FIRST METATARSOPHALANGEAL JOINT (MTP1) IS NOT THE MAIN LOCALIZATION OF GOUT AT DIAGNOSIS IN SUB-SAHARAN AFRICA}

F. Kemta Lekpa, M.S. Doualla, H. Namme Luma. Service de Médecine Interne, Douala General Hospital, Douala, Cameroon

Background: Numerous data in sub-Saharan Africa suggest that MTP1 is not the hallmark of gout (1-5).

Objectives: We carried out this study with the aim to determine the joint most involved at the time of diagnosis of gout in Cameroon.

Methods: We performed a cross-sectional study in all outpatients seen at the Rheumatology unit of the Douala General Hospital, Cameroon, between 2004 and 2014. We included patients with diagnosis of gout according to ACR criteria 1977.

The main characteristics of gout at diagnosis were collected, particularly the joints involved.

A $p<0.05$ was significant.

Results: At the end of this study, 511 patients ( 415 men and 96 women) with the diagnosis of gout were included. The mean age was $55.9 \pm 10.8$ years.

Joint pain $(n=508,99.4 \%)$ was the leading reason for consultation at the time of diagnosis. The knees $(n=300,62.6 \%))$, ankles $(n=187,39.0 \%)$ and MTP1 $(n=128$, $26.7 \%$ ) were the most affected joints. Table 1 presents the frequency of the joints affected, comparing our results with those of the other African series.

There was no difference between MTP1 and others joints location (particularly knees and ankles) according to age, sex, place of residence, duration of disease, uric acid level, and associated comorbidities ( $p>0.05$ ).

Conclusions: MTP1 is not the joint most involved at the time of diagnosis of gout in sub-Saharan Africa. Diagnosis of gout should be considered before any inflammatory knee and ankle pain in patients from sub-Saharan Africa. Genetic studies would provide a better understanding of this feature.

\section{References:}

[1] Kodio B, et al. Afr J Rheumatol. 2015 3(1): 22-26.

[2] Adelewo OO, et al. Afr J Rheumatol. 2014 1(2): 23-28.

[3] Singwé-Ngandeu M, et al. Mali Medical. 2009 24:17-20.
Table 1. Comparison of the most affected joints of the different African series*

\begin{tabular}{lcccccc}
\hline & $\begin{array}{c}\text { Our study } \\
\text { (Cameroon), } \\
2017\end{array}$ & $\begin{array}{c}\text { Kodio } \\
\text { (Mali), }\end{array}$ & $\begin{array}{c}\text { Adelowo } \\
\text { (Nigeria), }\end{array}$ & $\begin{array}{c}\text { Singwé- } \\
\text { Ngandeu } \\
\text { (Cameroon), } \\
2015\end{array}$ & $\begin{array}{c}\text { Mijiyawa } \\
\text { (Togo), } \\
2000\end{array}$ & $\begin{array}{c}\text { Cassim } \\
\text { (South Africa), } \\
1994\end{array}$ \\
\hline Number of patients & 511 & 100 & 146 & 139 & 160 & 107 \\
Sex (M/F) & $415 / 96$ & $45 / 55$ & $108 / 38$ & $131 / 8$ & $159 / 1$ & $93 / 14$ \\
Mean age & $55.9 \pm 11$ & 57 & $53.4 \pm 11$ & $55.7 \pm 10$ & 44 & $50.5 \pm 11.5$ \\
Joints involved (tender and/or stiffness), $\mathrm{n}(\%)$ & & & & \\
Knees & $300(62.6)$ & $92(92 \%)$ & $81(55.5)$ & $60(43.2)$ & $82(51.2)$ & $91(85)$ \\
Ankles & $187(39.0)$ & $45(45 \%)$ & $50(34.2)$ & $67(48.2)$ & $90(56.2)$ & $66(61.7)$ \\
1st MTP joint & $128(26.7)$ & $22(22 \%)$ & $21(14.4)$ & $65(46.8)$ & $100(62.5)$ & $80(74.8)$ \\
Wrists & $93(19.4)$ & $20(20 \%)$ & $14(9.6)$ & $30(21.6)$ & $30(18.7)$ & - \\
Elbows & $81(16.9)$ & $14(14 \%)$ & $12(8.2)$ & $20(10.7)$ & $35(21.9)$ & - \\
PIP joints & $56(11.7)$ & - & $13(8.9)^{\ddagger}$ & - & $15(9.4)^{\dagger}$ & - \\
MCP joints & $53(11.1)$ & - & - & - & - & - \\
Tarsal region & $50(10.4)$ & - & - & - & $50(31.2)$ & - \\
Shoulders & $49(10.2)$ & $6(6 \%)$ & - & - & - & - \\
Others MTP joints & $24(5.0)$ & - & - & - & $15(9.4)$ & - \\
DIP joints & $15(3.1)$ & - & - & - & - & - \\
Others & $30(6.3)$ & $1(1 \%)$ & - & $13(9.6)$ & $9(5.6)$ & - \\
\hline
\end{tabular}

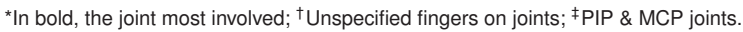

[4] Mijiyawa M, et al. Joint Bone Spine. 2000;67(5):441-5.

[5] Cassim B, et al. Ann Rheum Dis. 1994 Nov;53(11):759-62.

Disclosure of Interest: None declared

DOI: 10.1136/annrheumdis-2017-eular.6799

\section{THU0426 LONG-TERM ADHERENCE TO URATE-LOWERING THERAPY IN GOUT: DO NOT BLAME ON THE PATIENTS}

F. Perez-Ruiz, I. Urionagüena, S.P. Chinchilla. Rheumatology Division, Hospital Universitario Cruces and Biocruces Health Research Institute, Baracaldo, Spain

Background: adherence to urate-lowering treatment (ULT) in patients with gout is reported to be lower than $50 \%$ in the first year, below $20 \%$ at 2-year, and worse than in other chronic conditions such as hypertension, diabetes, or hyperlipidemia. Objectives: to evaluate adherence to ULT both overall and during follow-up, to compare it to the adherence to medications for associated comorbidities, and to explore potential causes for non-adherence to ULT.

Methods: transversal study of a nested cohort of patients in a gout clinic in the hospital setting who were scheduled for a follow-up visit during 6 consecutive months in 2016. General data of patients, along with variables related to gout and to comorbid conditions are systematically retrieved at first visit; prescribed ULT, doses, adherence, and serum urate levels were obtained during the follow-up visits. Adherence was retrieved as medication possession rate (MPR) according to pharmacy offices from government electronic databases (including $>98 \%$ of the general population). Also, MPRs of drugs prescribed for hypertension, diabetes (only oral), and hyperlipidemia were obtained; if more than one drug prescribed for any of the previous, the best adherence per comorbidity treatment was entered. Good adherence was considered as MPR $>80$ percent of that prescribed, target serum urate $(\mathrm{s} \cup A)$ as $<0.36 \mathrm{mmol} / \mathrm{L}$. Patients are educated at first visit and encouraged to be adherent from baseline through follow-up visits.

Results: adherence data were available from 209 patients who were scheduled for a follow-up visit during the observation period; $14(6.7 \%)$ patients did not atend the visit. This sample was formed by $90 \%$ male, only $55 \%$ had received ULT previous to first visit, median age was 65 years at follow-up visit, $47 \%$ and $44 \%$ showed poliarticular and tophaceous disease at baseline, respectively. MPR overall showed a median of $89 \%$ (IQR 79-94, N=209) for ULT (72\% had MPR $>80$ ), and $89 \%$ (IQR 81-94, $\mathrm{N}=119$ ), 88\% (IQR 79-94, $\mathrm{N}=65$ ), and $82 \%$ (IQR 77-93, N=28) for hypertension, hyperlipidemia, and diabetes respectively ( $<<0.05$ only for diabetes).

Adherence was lower for patients who did not attend the scheduled visit (median MPR $58 \%$ vs. $86 \%$, MPR $>80,21 \%$ vs. $75 \%, p<0.01)$. Adherence was lower during the first year $(80 \%, N=67)$ compared to 2 nd and 3 rd year period $(86 \%$, $\mathrm{N}=67$ ) or 4 year or over ( $89 \%, \mathrm{~N}=75)$. MPR $>80$ was $57 \%, 76 \%$ y $81 \%$ for the same periods of follow-up, respectively. Good adherence was associated to a rate of target serum urate of $90 \%$, compared to $72 \%$ for patients showing MPR $<80$. Male gender and un-attendance to scheduled visit were statistically associated to $\mathrm{MPR}<80$ in multivariate analysis, and numerically to increasing age and overall comorbidity.

Conclusions: adherence to ULT measured as MPRs in a cohort of educated patients is good, sustained during follow-up, and similarly good to that for comorbid conditions (hypertension, hyperlipidemia, and diabetes); therefore, we cannot blame poor adherence on the patients anymore. Targeting absenteeism could be an opportunity for further improvement.

Disclosure of Interest: F. Perez-Ruiz Consultant for: Amgen, Ardea, AstraZeneca, Gruenenthal, Menarini, Speakers bureau: AstraZeneca, Gruenenthal, Menarini, I. Urionagüena: None declared, S. Chinchilla: None declared DOI: 10.1136/annrheumdis-2017-eular.2994 